\title{
Word Searching in Document Images Using Word Portion Matching
}

\author{
Yue Lu and Chew Lim Tan \\ Department of Computer Science, School of Computing \\ National University of Singapore, Kent Ridge, Singapore 117543 \\ $\{$ luy, tancl\}@comp.nus.edu.sg
}

\begin{abstract}
An approach with the capability of searching a word portion in document images is proposed in this paper, to facilitate the detection and location of the user-specified query words. A feature string is synthesized according to the character sequence in the user-specified word, and each word image extracted from documents are represented by a feature string. Then, an inexact string matching technology is utilized to measure the similarity between the two feature strings, based on which we can estimate how the document word image is relevant to the user-specified word and decide whether its portion is the same as the user-specified word. Experimental results on real document images show that it is a promising approach, which is capable of detecting and locating the document words that entirely match or partially match with the user-specified word.
\end{abstract}

\section{Introduction}

With the rapid development of computer technology, digital documents have become popular for storage and transmission, instead of the traditional paper documents. The most widespread format for these digital documents is the text in which the characters of the documents are represented by the machine-readable codes(e.g. ASCII codes). The majority of the newly generated documents are in the text format. On the other hand, to make billions of volumes of traditional paper documents available and accessible in the format of digital domain, they are scanned and converted to digital images by the digitization equipment.

Although the technology of Optical Character Recognition(OCR) may be utilized to automatically transfer the digital images of these documents to their machine-readable text format, the OCR has still its inherent weaknesses in the recognition ability, especially for the poor quality document images. Generally speaking, manual correction/proofing of the OCR results is usually unavoidable, which is typically not cost effective for transferring a huge amount of paper documents to their text format. Moreover, the technology of layout analysis is still immature for the documents with complicated layouts. As a result, storing these documents in the image format has become an alternative way in many cases. Nowadays, many digital documents are in the image format. It is therefore 
of significant meaning to study the strategies of retrieving information from these document images.

Motivated by the fact that the OCR accuracy requirements for information retrieval are considerably lower than for many document processing applications, the methods with the ability of tolerating recognition errors of OCR have been researched $[12$. However, the layout analysis and character segmentation are unavoidable in these OCR-based methods. In recent years, a number of attempts have been made to avoid the use of OCR for retrieving information from document images 34 .

Searching and locating a user specified keyword in the image format documents has its practical value for document information retrieval. For example, by using this technique the user can locate a specified word in the document images without the requirement of OCRing the entire document.

In the case where there are a large number of image documents in the Internet, the user has to download each one to see its contents before knowing whether the document is relevant to his interest. The image based keyword searching technology is capable of notifying the user whether a document image contains words of interest to him, and indicating which documents are worth downloading, prior to transmitting the document images through the Internet.

Some image-based approaches to searching keyword in handwritten 56 and printed [7 8 1910 10 11] documents have been reported in the past years. To avoid the difficulties of segmenting connected characters in a word image, segmentationfree methods were applied in these approaches. For example, the hidden Markov model and Viterbi dynamic programming algorithm are widely used to recognize keywords. However, these approaches have their disadvantages. Although DeCurtins' approach 7] and Chen's approach 910 are capable of searching a word portion, it can only process the predefined keywords, and the pre-training procedure is unavoidable. In our previous word searching system 11, a weighted Hausdorff distance is used to measure the dissimilarity between word images. Although it can cope with any words the user specified, it cannot deal with the problem of portion matching. As a result, the approach is not able to search the word "images" while the user keys in a word "image", because they are considered as different words from the image standpoint.

In this paper, we propose an approach based on partial word image matching, which has the ability of searching the words in the document images if their portions match the user-specified words. For example, it can detect the words such as "knows" and "unknown" while the user keys in the query word "know". A feature string is synthesized according to the the character sequence in the user-specified word, and each word image extracted from documents are represented by a feature string. Then, an inexact string matching technology is utilized to measure the similarity between the two feature strings. Based on the similarity measurement we can estimate how the document word image is relevant to the user specified word. Experiments have been conducted to detect and locate the user-specified words in real document images. The results show that it is a promising approach with the capability of word portion searching. 


\section{Feature String}

To search a user-specified word in a document image, the feature string of the user-specified word is first generated by synthesizing the feature of each character in the word. Then it is matched with the feature string of each word image bounded in the document. According to the matching scoring the corresponding word image is decided whether its portion is the same as the user-specified word.

To extract the feature string of a word image, we scan the word image column by column, and give each column a code. Different code represents different feature as indicated below:

$\&$ : there is no image pixel in the column. It corresponds to the blank space between characters generally.

1: upper long vertical strokes. There is only one vertical stroke in the column. Its length is greater than the x-height of the word image, and it is located above the base line.

2: lower long vertical strokes. There is only one vertical stroke in the column. Its length is greater than the $\mathrm{x}$-height of the word image, and it is located below the mean line.

3: vertical strokes. There is only one vertical stroke in the column, and its length is shorter than the $\mathrm{x}$-height of the word image but longer than half of the $\mathrm{x}$-height of the word image.

4: short vertical strokes at the upper-side of the mid-zone. There is in the column only one vertical stroke whose length is shorter than half of the x-height of the word image, and it is located at the upper-side of the mid-zone.

5: short vertical strokes at the lower-side of the mid-zone. There is in the column only one vertical stroke whose length is shorter than half of the x-height of the word image, and it is located at the lower-side of the mid-zone.

6: there are two strokes in the scanned column.

7: there are three or more strokes in the scanned column.

Then, the adjacent neighboring features with the same codes are merged to one code. Figure 1 shows an example of extracting the feature codes from the word image "able".

To achieve the ability of dealing with different fonts, the features used to represent a character should be independent of typefaces. It is a basic necessity to avoid the effect of serif in a printed character. Our observation found that a feature code produced by serif can be eliminated by analyzing its preceding and succeeding features. Fox example, a feature ' 5 ' in a feature subsequence ' $35 \&$ ' is normally generated by a right-side serif of characters such as 'a', 'd', 'h', 'm', 'n', ' $u$ ', etc. Therefore, we can remove straightforward the feature ' 5 ' from a feature subsequence '35\&', as shown in Figure 1.

Based on the feature extraction described above, we can give each character a feature sequence. For example, the feature sequence of character ' $b$ ' is ' 163 ', and that of character ' $p$ ' is ' 263 '. The feature string of a user specified word can be generated by synthesizing the feature sequence of each characters in the word and inserting a special feature ' $\&$ ' among them to identify a spacing gap. 


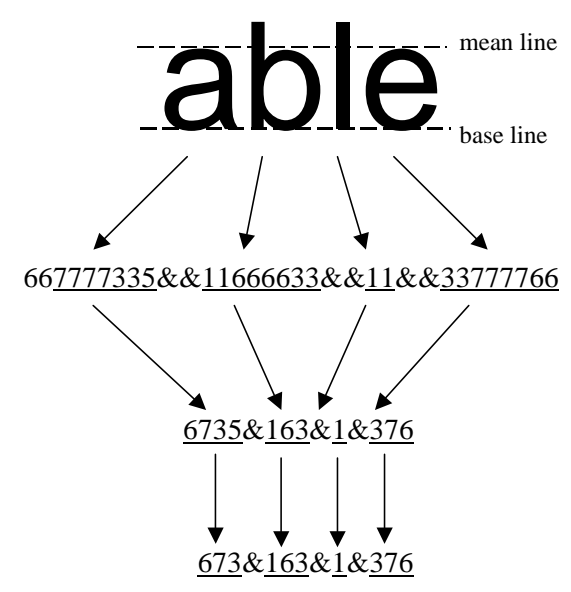

Fig. 1. Feature string

\section{$3 \quad$ Inexact Feature Matching}

Based on the above processing, each word image is described by a feature string. The procedure of searching word image has become measuring the similarity between the string $S=\left[S_{1}, S_{2}, \ldots, S_{n}\right]$ representing the features of the user-specified word and the string $T=\left[T_{1}, T_{2}, \ldots, T_{m}\right]$ representing the features of the word image extracted from the document. Matching word portion becomes evaluating the similarity between the feature string $S$ with the sub-sequence of the feature string $T$.

In a word image, it is common that two or more adjacent characters are connected with each other, which is possibly caused by low scanning resolution or poor printing quality. This will result in the deletion of the feature ' $\&$ ' in the corresponding feature string, compared to its standard feature string. Moreover, noise effect will undoubtedly produce the substitution or insertion of features in the feature string of the word image. The deletion, insertion and substitution are very similar to the course of evolutionary mutations of DNA sequences in molecular biology.

Drawing inspiration from the alignment problem of two DNA sequences, we apply the technology of inexact string matching to evaluate the similarity between the feature string of the user-specified word and that of the word image extracted from document. From the standpoint of string alignment 12, two opposing features that mismatch correspond to a substitution; a space contained in the first string corresponds to an insertion of the opposing feature into the first string; and a space in the second string corresponds to a deletion of the opposing features from the first string. The insertion and deletion are the reverse of each other. A dash( "-") is therefore used to represent a space feature inserted into the corresponding positions in the strings for the situation of deletion. Notice 
that we use 'spacing' to represent the gap in the word image, whereas we use 'space' to represent the inserted feature in the feature string. Their concepts are completely different.

Definition 1 For a string $S$ of length $n$ and a string $T$ of length $\mathrm{m}, V(i, j)$ is defined to be the similarity value of the prefixes $\left[S_{1}, S_{2}, \ldots, S_{i}\right]$ and $\left[T_{1}, T_{2}, \ldots, T_{j}\right]$. The similarity of $S$ and $T$ is precisely the value $V(n, m)$.

Definition 2 Let $\Sigma=\{\&, 1,2,3,4,5,6,7\}$ be the feature set of the strings $S$ and $T$, and let $\Sigma^{\prime}$ be $\Sigma$ with the added feature "-" denoting a space. Then, for any two features $x, y$ in $\Sigma^{\prime}, \sigma(x, y)$ denotes the score obtained by aligning the feature $x$ against the feature $y$.

The similarity of two strings $S$ and $T$ can be computed by dynamic programming with recurrences. The base conditions are :

$$
\forall i, j \text { : }
$$

$$
\left\{\begin{array}{l}
V(i, 0)=0 \\
V(0, j)=0
\end{array}\right.
$$

The general recurrence relation is: for $1 \leq i \leq n, 1 \leq j \leq m$ :

$$
V(i, j)=\max \left\{\begin{array}{l}
0 \\
V(i-1, j-1)+\sigma\left(S_{i}, T_{j}\right) \\
V(i-1, j)+\sigma\left(S_{i},-\right) \\
V(i, j-1)+\sigma\left(-, T_{j}\right)
\end{array}\right.
$$

The zero in the above recurrence implements the operation of 'restarting' the recurrence, which can make sure that the unmatched prefixes are discarded from the computation.

We choose the matching scoring between any two features $x$ and $y$ in $\Sigma^{\prime}$ as:

$$
\sigma(x, y) \begin{cases}\geq 0 & \text { if } x \text { is same as/similar to } y \\ \leq 0 & \text { else }\end{cases}
$$

In the experiments, the scoring of matching any feature in $\Sigma$ with the space '-' is defined as:

$$
\sigma\left(S_{k},-\right)=\sigma\left(-, T_{k}\right)=-2 \quad \text { for } S_{k}, T_{k} \in \Sigma
$$

while the scoring between any feature in $\Sigma$ is defined in Table 1.

The problem can be evaluated systematically using a tabular computation. In this approach, a bottom-up approach is used to compute $V(i, j)$. We first compute $V(i, j)$ for the smallest possible values for $i$ and $j$, and then compute the value of $V(i, j)$ for increasing values of $i$ and $j$. This computation is organized with a dynamic program table of size $(n+1) \times(m+1)$. The table holds the values of $V(i, j)$ for the choices of $i$ and $j$ (see Table 2). Note that the string $S$ corresponds to the horizontal axis of the table, while the string $T$ corresponds to the vertical axis. The values in row zero and column zero are filled in directly from the base conditions for $V(i, j)$. After that, the remaining $n \times m$ sub-table 
Table 1. Scoring between features

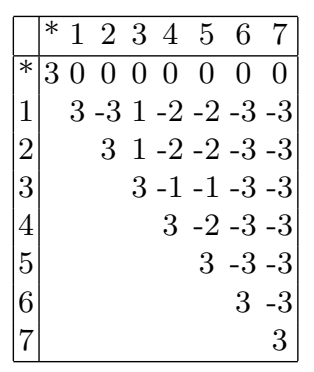

is filled in one row at a time, in the order of increasing $i$. Within each row, the cells are filled in the order of increasing $j$.

The following pseudo code describes the algorithm:

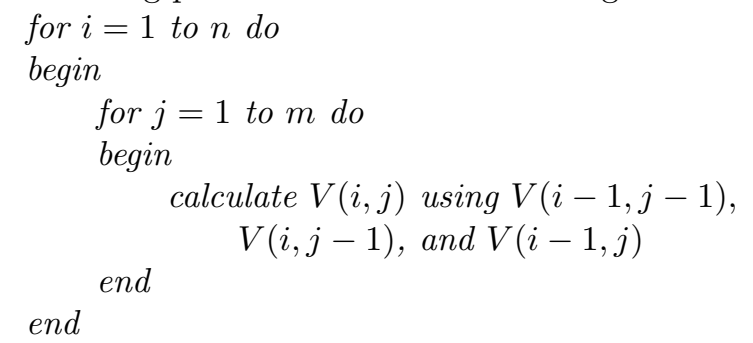

The entire dynamic programming table for computing the similarity between a string of length $n$ and a string of length $m$, can be obtained in $O(n m)$ time, since only three comparisons and arithmetic operations are needed per cell.

Table 2 illustrates the scoring table computing from the feature strings of the word image "enabled" and the user specified word "able". It can be seen that the maximum scoring achieved in the table corresponds to the matching of character sequence "able" in the word "enabled".

Then, the maximum scoring is normalized as:

$$
\text { score }=\max _{\forall i, j} V(i, j) / V_{s}^{*}(n, n)
$$

where $V_{s}^{*}(n, n)$ is the matching score between the string $S$ and itself.

If the score is greater than a predefined threshold $\delta$, then we recognize that a portion of the word image matches the user-specified word.

\section{Experiments}

To verify the validity of the proposed approach of word portion matching, we use it to detect and locate the user-specified words in the real document images. The document images are selected from the scanned books and students' theses that are provided by the Central Library of the National University of Singapore. 
Table 2. Scoring table for computing $V(i, j)$

\begin{tabular}{|c|c|c|c|c|c|c|c|c|c|c|c|c|c|c|c|c|}
\hline & \multicolumn{5}{|c|}{ a } & \multicolumn{3}{|c|}{ b } & \multicolumn{3}{|c|}{ I } & \multicolumn{3}{|c|}{ e } & \\
\hline & & - & 6 & 7 & 3 & \& & 1 & 6 & 3 & $\boldsymbol{d}$ & 1 & \& & 3 & 7 & 6 & \\
\hline \multirow{6}{*}{ e } & - & 0 & 0 & 0 & 0 & 0 & 0 & 0 & 0 & 0 & 0 & 0 & 0 & 0 & 0 & \\
\hline & 3 & 0 & 0 & 0 & 3 & 1 & 1 & 0 & 3 & 1 & 1 & 0 & 3 & 1 & 0 & \\
\hline & 7 & 0 & 0 & 3 & 1 & 3 & 1 & 0 & 1 & 3 & 1 & 1 & 1 & 6 & 4 & \\
\hline & 6 & 0 & 3 & 1 & 0 & 1 & 0 & 4 & 2 & 1 & 0 & 1 & 0 & 4 & 9 & \\
\hline & $\&$ & 0 & 1 & 3 & 1 & 3 & 1 & 2 & 4 & 5 & 3 & 3 & 1 & 2 & 7 & \\
\hline & 3 & 0 & 0 & 1 & 6 & 4 & 4 & 2 & 5 & 4 & 6 & 4 & 6 & 4 & 5 & \\
\hline \multirow[t]{4}{*}{$\mathrm{n}$} & 4 & 0 & 0 & 0 & 4 & 6 & 4 & 2 & 3 & 5 & 4 & 6 & 4 & 3 & 3 & \\
\hline & 5 & 0 & 0 & 0 & 3 & 4 & 7 & 5 & 5 & 3 & 6 & 4 & 9 & 7 & 5 & \\
\hline & $\&$ & 0 & 0 & 0 & 1 & 6 & 5 & 7 & 5 & 8 & 6 & 9 & 7 & 9 & 7 & \\
\hline & 6 & 0 & 3 & 1 & 0 & 4 & 3 & 8 & 6 & 6 & 5 & 7 & 6 & 7 & 12 & \\
\hline \multirow[t]{4}{*}{ a } & 7 & 0 & 1 & 6 & 4 & 2 & 1 & 6 & 5 & 6 & 4 & 5 & 4 & 9 & 10 & \\
\hline & 3 & 0 & 0 & 4 & 9 & 7 & 5 & 4 & 9 & 7 & 7 & 5 & 8 & 7 & 8 & \\
\hline & $\&$ & 0 & 0 & 2 & 7 & 12 & 10 & 8 & 7 & 12 & 10 & 10 & 8 & 8 & 7 & \\
\hline & 1 & 0 & 0 & 0 & 5 & 10 & 15 & 13 & 11 & 10 & 15 & 13 & 11 & 9 & 7 & \\
\hline \multirow[t]{3}{*}{ b } & 6 & 0 & 3 & 1 & 3 & 8 & 13 & 18 & 16 & 14 & 13 & 15 & 13 & 11 & 12 & \\
\hline & 3 & 0 & 1 & 0 & 4 & 6 & 11 & 16 & 21 & 19 & 17 & 15 & 18 & 16 & 14 & \\
\hline & $\&$ & 0 & 0 & 1 & 2 & 7 & 9 & 14 & 19 & 24 & 22 & 20 & 18 & 18 & 16 & \\
\hline \multirow[t]{3}{*}{ I } & 1 & 0 & 0 & 0 & 2 & 5 & 10 & 12 & 17 & 22 & 7 & 25 & 23 & 2 & 19 & \\
\hline & $\&$ & 0 & 0 & 0 & 0 & 5 & 8 & 10 & 15 & 20 & 25 & 30 & 28 & 26 & 24 & . \\
\hline & 3 & 0 & 0 & 0 & 3 & 3 & 6 & 8 & 13 & 18 & 23 & 28 & 33 & 3 & 29 & scoring \\
\hline \multirow[t]{4}{*}{ e } & 7 & 0 & 0 & 3 & 1 & 3 & 4 & 6 & 11 & 16 & 21 & 26 & 31 & 36 & 34 & \\
\hline & 6 & 0 & 3 & 1 & 0 & 1 & 2 & 7 & 9 & 14 & 19 & 24 & 29 & 34 & 39 & \\
\hline & $\&$ & 0 & 1 & 3 & 1 & 3 & 1 & 5 & 7 & 12 & 17 & 22 & 27 & 32 & 37 & \\
\hline & 3 & 0 & 0 & 1 & 6 & 4 & 4 & 3 & 8 & 10 & 15 & 20 & 25 & 30 & 35 & \\
\hline \multirow[t]{2}{*}{ d } & 6 & 0 & 3 & 1 & 4 & 6 & 4 & 7 & 6 & 8 & 13 & 18 & 23 & 28 & 33 & \\
\hline & 1 & 0 & 1 & 0 & 2 & 4 & 9 & 7 & 8 & 6 & 11 & 16 & 21 & 26 & 21 & \\
\hline
\end{tabular}

A connected component detecting algorithm is applied to identify all of the connected components in the document image first. The positional relations among the connected components are then utilized to bound the word images. The feature string of each word image is extracted for matching with the feature string of the user-specified word.

When a user keys in a specified word, the system generates its corresponding feature string according to the character sequence. The feature string is matched with each feature string of document words to measure the similarity between them. Figure 2 demonstrates one example, in which the words "processing" and "processed" are detected and located successfully in the document image within the bounding rectangles while the user keys in the word "process" for searching. Another example is given in Figure 3, in which the words "enabled" and "unable" are detected and located in the document image while the user inputs the word "able" for searching.

To evaluate the performance of the system, 25 images of scanned books and 324 images of scanned students' theses are included in the test. 100 words are selected to search their corresponding words and variations from the document images. The system achieves an average precision ranging from $91.57 \%$ to $99.21 \%$ and an average recall ranging from $87.38 \%$ to $97.12 \%$ depending on different threshold $\delta$, as shown in Table 3 ,

A lower $\delta$ results in higher recall but at the expense of lower precision. The reverse is true for a higher $\delta$. Thus, if the goal is to retrieve as many relevant 
Table 3. Performance vs. Threshold $\delta$

\begin{tabular}{|c|c|c|c|c|c|}
\hline Threshold $\delta$ & 0.75 & 0.8 & 0.85 & 0.9 & 0.95 \\
\hline Recall & $97.12 \%$ & $95.23 \%$ & $91.52 \%$ & $89.26 \%$ & $87.38 \%$ \\
\hline Precision & $91.57 \%$ & $92.29 \%$ & $94.60 \%$ & $98.73 \%$ & $99.21 \%$ \\
\hline
\end{tabular}

words as possible, then a lower $\delta$ should be set. On the other hand, for low tolerance to precision, then $\delta$ should be raised.

Experiments found that the present approach is able to deal with most commonly used fonts, but it cannot handle italic fonts. This is caused by the shortcoming of the features used to represent the word image, in which the features are obtained by vertically scanning the image column by column.

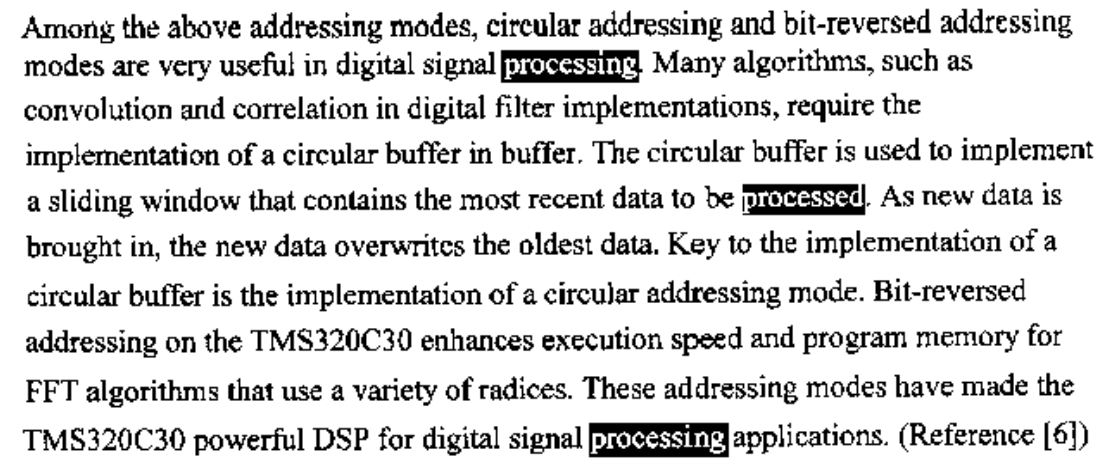

Fig. 2. Result of searching word "process"

\section{Conclusions}

We proposed in this paper an approach for word portion searching based on the technique of inexact matching. The user specified word and the word image extracted from documents are represented by two feature strings first. Then, an inexact string matching technique is utilized to measure the similarity between the two feature strings. The present approach has the ability to search words in document images, whose portions match with the user-specified words. The experimental results show that it is a promising approach for word image searching.

However, the present approach cannot handle the italic word image due to the inherent shortcoming of the feature string used to represent the word image. Further work is to overcome the limitation by using a new feature string which is applicable to all typefaces. 
July 5.-We revisited Shang-hae : though the wind was unfavourable, the tide enabled us to reach the place at halfpast seven o'clock. We took up our quarters at the temple of the queen of heaven, where the crowds gathered around us again. I began with distributing the Scriptures to them, as the best means to promote their eternal happiness. They seized them eagerly from my hands, and immediately retired to read them leisurely.

Confounded at our unceremonious visit, the mandarins came in great haste to the temple. They were at this time more humble and yielding than before, yet they had stuck up two outrageous proclamations, which I immediately copied. They also attempted to prevent us from going into the city, but we passed through another gate, which they were unable to shut. We now bought large quantities of silk, and some trifles, to which they at first made great objections, but very soon granted to us the liberty of

Fig. 3. Result of searching word "able"

In addition, from the analysis of recall, it has been found that most of the missing words are caused by the incorrect word bounding(fragmentation mostly). It is therefore necessary to improve the word bounding performance of the system.

Acknowledgements. This project is supported by the Agency for Science, Technology and Research, and Ministry of Education of Singapore under research grant R255-000-071-112/303.

\section{References}

1. Ishitani, Y.: Model-based Information Extraction Method Tolerant of OCR Errors for Document Images. In: Proc. of the Sixth International Conference on Document Analysis and Recognition, Seattle, USA (2001) 908-915

2. Ohtam, M., Takasu, A., Adachi, J.: Retrieval Methods for English Text with Misrecognized OCR Characters. In: Proc. of the Fourth International Conference on Document Analysis and Recognition, Ulm, Germany (1997) 950-956

3. Doermann, D.: The Indexing and Retrieval od Document Images: A Survey. Computer Vision and Image Understanding, Vol.70, No.3 (1998) 287-298 
4. Tan, C. L., Huang, W. H., Yu, Z., Xu, Y.: Imaged Document Text Retrieval without OCR. IEEE Trans. Pattern Analysis and Machine Intelligence, to appear

5. Manmatha, R., Han C., Riseman, E. M.: Word Spotting: A New Approach to Indexing Handwriting. In: Proc. of the International Conference on Computer Vision and Pattern Recognition (1996) 631-637

6. Syeda-Mahmood, T.: Indexing of Handwritten Document Images. In: Proc. of the Workshop on Document Image Analysis. San Juan, Puerto Rico (1997) 66-73

7. DeCurtins, J., Chen, E.: Keyword Spotting via Word Shape Recognition. In: Vincent, L. M., Baird, H. S. (eds.) Proceedings of SPIE, Document Recognition II, Vol.2422, San Jose, California (1995) 270-277

8. Kuo, S., Agazzi, O. F.: Keyword Spotting in Poorly Printed Documents Using Pseudo 2-D Hidden Markov Models. IEEE Trans. Pattern Analysis and Machine Intelligence vol.16, No.8(1994) 842-848

9. Chen, F. R., Wilcox, L. D., Bloomberg, D. S.: Word Spotting in Scanned Images Using Hidden Markov Models. In: Proc. of the International Conference on Acoustics, Speech, and Signal Processing, Vol.5(1993) 1-4

10. Chen, F. R., Wilcox, L. D., Bloomberg, D. S.: Detecting and Loacting Partially Specified Keywords in Scanned Images Using Hidden Markov Models. In: Proc. of the International Conference on Document Analysis and Recognition (1993) $133-138$

11. Lu, Y., Tan, C. L., Huang, W., Fan, L: An Approach to Word Image Matching Based on Weighted Hausdorff Distance. In: Proceedings of the Sixth International Conference on Document Analysis and Recognition, Seattle, USA (2001) 921-925

12. Gusfield, D.: Algorithms on Strings, Trees, and Squences. Combridge University Press (1997) 